\title{
Clinical and academic recommendations for primary dental care prosthodontics
}

\author{
Ryan C. Olley ${ }^{* 1,3}$ and Peter M. Frost BEM ${ }^{2,3}$
}

\section{Key points}

The need for clinical research to improve oral and dental health is reviewed.
Challenges and needs in primary dental care are discussed.
Clinical and academic questions and recommendations are developed to help drive improvements in oral and dental health, with relevance to prosthodontics, in primary dental care.

\begin{abstract}
Prosthodontic practice includes a large proportion of dental care provision, mostly undertaken in primary dental care (PDC) in the UK. However, there is limited research evidence in prosthodontic practice, necessary to tackle key questions to improve oral and dental health. In addition, many overarching challenges exist within the NHS system relevant to prosthodontics. For example, demands on healthcare services from increasing patient expectations, the ever-ageing and growing population, risks of litigation, availability of appropriate care, difficulties with access to care, rapidly emerging treatments and technologies, and ongoing needs for education. This article develops clinical and academic recommendations to improve oral health improvements in PDC prosthodontics, further to recommendations determined by the James Lind Alliance, a nonprofit making initiative to tackle treatment 'uncertainties' (http://www.jla.nihr.ac.uk/), and the overarching challenges, which are mentioned above. Considerable clinical and academic author experiences from primary, secondary and tertiary care as well as education and research in prosthodontics are employed. We consider management of networks involving patients, carers, healthcare professionals, industry and academics, in order to increase access to improve oral and general health and help drive dental research, education and communication. We investigate how to manage dental disease and intervene to improve the longevity of restorations and the dentition. Finally, we review how rapidly developing digital technologies can best be utilised, in conjunction with industry, in order to improve oral health.
\end{abstract}

\section{Need for appropriate research to improve oral and dental health}

Tackling treatment uncertainties together

Reducing disease and improving health are roles of all healthcare professionals. As such, we rely on high quality, relevant evidence. ${ }^{1}$ When searching the literature for answers to healthcare interventions, the validity of research may be limited by a lack of focus on specific areas relevant to clinical practice, poor quality evidence or scarcity of investigation. In order to better inform healthcare research agendas, the James Lind Alliance (JLA) was

${ }^{1}$ Clinical Specialist and Academic in Prosthodontics, West Mersea Dental Practice, Colchester, C05 8RA, UK; ${ }^{2}$ Clinical Specialist and Senior Clinical Teacher, 178 Peckham Rye Lane, London, SE22 9QA, UK; ${ }^{3}$ King's College London, Faculty of Dentistry, Oral and Craniofacial Sciences, UK. ${ }^{*}$ Correspondence to: Dr Ryan Olley

Email: ryan.2.olley@kcl.ac.uk

Accepted 3 July 2019

https://doi.org/10.1038/s41415-019-0785-6 established in 2004 as a non-profit making initiative to tackle treatment 'uncertainties.' The JLA defines uncertainties as known unknowns, ie relating to the effects of treatment. Specifically, they include questions about treatments such as options or methods of care and diagnostic testing, which cannot be answered by existing research. This may be due to non-existent or a lack of up-to-date systematic reviews, or alternatively up-todate systematic reviews that demonstrate uncertainty about treatments. ${ }^{2}$

It is useful to reflect on research agendas in the context of the key values of our National Health Service (for NHS England) published in June $2014,{ }^{3}$ following the Health and Social Care Act 2012. ${ }^{4}$ The focus of this document, perhaps surprisingly as late as in 2012 , was on increasing public and patient involvement in improving healthcare. The aims of the JLA reflect this leaning, by including patient ('anyone' who has received healthcare), carer, clinician and academic groups 'together' on an equal footing through Priority Setting
Partnerships (PSPs). These partnerships are designed to address the mismatch between what researchers may want to research and practical information on what patients and clinical healthcare professionals need on a day-to-day basis in order to prioritise future research. ${ }^{5}$ This helps increase the relevance of research to inform education and clinical practice. $^{2}$

\section{Clinical research need in primary dental} care

In dentistry in the UK, most healthcare delivery occurs in primary dental care (PDC). However, there are often few large scale clinical trials investigating interventions to improve oral health, fragmentation of research in these areas, and lack of ambitious, collaborative and multi-centre research. ${ }^{6}$ Sadly, this is often due to poor funding and a need for sizeable resources to enable high quality research. When funding is available, oral and dental research may then not be prioritised on 'key' areas as described above. ${ }^{6}$ 
In 2017, an oral and dental PSP coordinated by the National Institute for Health Research Evaluation, Trials and Studies Coordinating Centre (NETSCC) was established. Ten priorities were published in December 2018. ${ }^{7}$ Table 1 further develops these into clinical and academic suggestions, which will be discussed in context below.

\section{Clinical challenges and need within primary dental care}

In 2008, when NHS healthcare focused toward high quality for all, ${ }^{8}$ challenges or risks to healthcare services were documented. These included changes in population demographics (ie, more patients) and concomitant transformations in disease patterns (as a result of the ever-ageing population), increasing demands on NHS services, a rise in patient expectations, advances in treatment and digital technologies, and the implementation and costs involved. More than ten years on, many of these challenges continue to exist. ${ }^{9,10}$ In addition, problems with access, rationing of NHS services, longer waiting times, stress on primary and community services, and delayed transfers continue to be reported. ${ }^{9}$

Recent reports, among newly qualified dental graduates, describe their reluctance to work within $\mathrm{PDC}^{11}$ and reported lack of clinical experience. ${ }^{10}$ There are limited studies investigating recent graduate destinations, but the British Dental Journal in 2018 described an overall dental recruitment crisis due to litigation, regulation and lack of resources not keeping pace with rapidly advancing technologies. ${ }^{12}$ The reluctance is further understandable from the rising risk of superfluous litigation ${ }^{10}$ fuelled by media outlets and legal firms, and hesitancy of legal representation by indemnity companies. ${ }^{11}$ A recent survey showed that $68 \%$ of dental practices were struggling to recruit. ${ }^{13}$ This is not good 'value' for the UK health service, which contributed training. It is suggested that dentists confidence could be improved by better preparing dental healthcare professionals throughout their career in communication, complaints handling, stress, management and financial planning. ${ }^{11}$

In addition, there is no universal system to make secondary care services available to NHS PDC. The vast proportions of specialist services are limited to teaching hospitals. This is unsuitable for access to complex care. Earlier studies from Northern England in $2005^{14}$ demonstrated a strong demand for specialist restorative services among dental practitioners, in particular for mono-specialist services (ie, prosthodontists, periodontists and endodontists). Reported barriers to specialist NHS services, including waiting times, excessive travel distances ${ }^{14}$ as well as costs to fund these services, continue. In another study in North East England in 2007 (with a focus on periodontology services), the difficulty managing specialist care under the current remuneration system, reluctance for general dental practitioners to manage advanced disease and a perceived potential for medico-legal consequences were all strong drivers for referrals. ${ }^{15}$ However, similar challenges included large travel distances to specialist referral centres and perceived costs of treatment (as most specialist referrals are undertaken privately). ${ }^{15}$ Despite this, there are currently few or no opportunities for dental specialists to contribute to the NHS system in practice. The same is often true for hygienists and therapists. A need exists to provide local secondary care access through the NHS. Private only care can increase inequalities especially in disadvantaged group $\mathrm{s}^{16}$ and therefore enabling private influence is not entirely advantageous to improving health equality.

Finally, a number of corporate clinical networks have been established for PDC over the past ten years, but there is a need for long-term data on the clinical effectiveness of services towards improving oral health in the UK. ${ }^{17}$ In a recent study, problems with autonomy, decision making capabilities and control were reported by corporate associates in PDC, in contrast to independent associates. ${ }^{17}$ Focus on targets and quantity rather than equitable provision of service and financial cuts are reported as concerns. ${ }^{17}$

\section{Clinical and academic recommendations for prosthodontics}

\section{Definition of prosthodontics}

Prosthodontics arose as a dental speciality due to increased litigation, patient expectations and the rapid advancements in materials and technologies in prosthetic dentistry. ${ }^{18}$ The current (2017) definition of prosthodontics from the Glossary of Prosthodontic terms is 'the dental speciality pertaining to the diagnosis, treatment planning, rehabilitation, and maintenance of the oral function, comfort, appearance, and health of patients with clinical conditions associated with missing or deficient teeth and/or maxillofacial tissues by using biocompatible substitutes. ${ }^{19}$ This definition is a broad approach to care. Prosthodontics involves both the art of a practical craft skill to reconstruct teeth, as well as underlying science. The majority of adult patients (edentulous, partially dentate and dentate) attending PDC in the NHS will therefore receive some form of prosthodontics. Furthermore, in May 2019, there were only 439 dentists on the General Dental Council prosthodontic specialist list, but 41,097 registered dentists and a further 71,609 dental care professionals. ${ }^{18}$ Therefore, non-specialist providers within PDC provide the majority of prosthodontic care and even following specialist care, a need exists for maintenance of most prostheses locally and in PDC. Therefore, the relevance of ongoing professional education cannot be understated for increasingly complex care, delivered by the dental team, which will be discussed further below.

As part of their overall care, patients may also require management by allied disciplines within restorative dentistry, including periodontology and endodontology, or other specialities including orthodontic, oral surgery and/or special care, to name a few.

\section{Questions and recommendations for prosthodontics}

Based on the challenges and needs discussed above, significant changes to support clinicians, services and patients are required for PDC. Outlined below are three clinical and academic questions and recommendations for prosthodontics in UK PDC. These are further summarised in Table 1 under the headings 'access', 'oral and general health', 'communication', 'dental disease' and 'digital'.

\section{How can we create and manage clinical networks to improve oral health, education and research for prosthodontics in PDC?}

Enabling quality in clinical dental practice through access to equitable services and based on patient experience has been suggested. ${ }^{20}$ In this regard, Managed Clinical Networks (MCNs) have been developed as 'linked groups of health professionals and organisations from primary, secondary and tertiary care working in a coordinated manner, unconstrained by existing professional and organisational boundaries to ensure equitable provision of high quality, clinically effective services. ${ }^{21}$ MCNs are necessary to manage an unrealistic 


\section{Clinical and/or academic suggestions to improve oral and dental} health for prosthodontics in PDC

How to manage MCNs to enable local complex care delivery and reduce inequalities?

A Access How to manage data to inform oral and dental health? Who to fund oral rehabilitation for clinical services?

How to develop interactions between dental health professionals in prosthodontic PDC and other healthcare professionals to improve general health? How are the

B

Oral and

General Health How to effectively monitor oral health and detect oral cancer?

How to maintain prostheses (for example, following cancer therapy) to ensure access locally? Which tools to screen disease and improve access?

\section{Examples of interventions (non-exhaustive)}

- Ensure provision of care through NHS as well as private networks.

- Investigate toolkits to identify patients at risk who require referral to a dental professional ${ }^{41}$ and health promotion strategies. ${ }^{26,41}$

- Audit disease prevention, for example prescription of high strength fluoride dentifrice for high caries risk patients.

- Investigations into rehabilitations such as the shortened dental arch to restore aesthetics and function, as priorities for access to funded services in PDC. ${ }^{33}$

- In edentulous individuals with implant over-dentures, liaison with dieticians and general medical practitioners may benefit general health. ${ }^{42}$

- In dentate patients with ongoing acid reflux, symptoms may be 'silent'. ${ }^{43} \mathrm{~A}$ small proportion may have Barrett's oesophagus ${ }^{43}$ and potential for development of oesophageal adenocarcinoma. ${ }^{44}$ Prosthodontic signs often include active pathological wear affecting

the palatal surfaces of maxillary anterior teeth and a need sometimes exists for liaison with medical practitioners

- After specialist care, training for ongoing local maintenance of prostheses is required in PDC.

- Develop mentorship programmes;

How to improve staff communication networks for multi- - Ensure appropriate education on communication for often multi-disciplinary specialist disciplinary care in prosthodontics?

Communication How to improve communication for patient consent in prosthodontics? care;

- Consider needs for consent. Prosthodontics as an art form may require visual as well as verbal and written forms of consent.

- Tooth wear and dentine hypersensitivity are increasing. ${ }^{25}$ This could lead to treatment complexities in an ageing dentition, ${ }^{25}$ which is a reason for restorative care interventions. ${ }^{45}$ There is a need for ongoing, large-scale, studies, which investigate the benefits of various interventions on prevention of dental disease and investigate the longevity of emerging materials, to inform prosthodontic management. A need also exists to better utilise commercial funding routes.

- Funded managed networks to maintain complex prosthetic rehabilitations long term.

- Feasibility studies into the development and use of digital networks involving industry, multi-centre and multi-partner collaborations with quality outcome measures for oral health;

- Develop clinical academic networks to inform digital workflow;

- Utilise digital technologies to provide a non-exhaustive resource of constant practice and updating, to enhance psychomotor and haptic feedback skills necessary for craft-based prosthodontic learning. existing dental remuneration system, problems with access to care and patient flow, financial restraints and limited resources for specialist services, ${ }^{10}$ as discussed above. Some MCNs have been developed, ${ }^{22}$ but there exists a need for prosthodontic MCNs. Patient flow, a priority for the Care Quality Commission, can be prioritised through MCNs and administration between centres more effectively streamlined. ${ }^{22}$

Networks require appropriate leadership whilst integrating with other healthcare professionals, avoiding financial targets and achieving the best outcomes to benefit patients. ${ }^{23}$ Clinical leadership can be the role of a specialist or consultant in the speciality concerned, through either tier 2 or tier 3 pathways. Academic leadership will prioritise research based on oral and dental needs. Therefore, an alternative and perhaps more realistic term for MCNs would be Managed Clinical Academic Networks (MCANs).
Prosthodontic clinical management is becoming more complex, with patients living longer and requiring ever more complex interventions to maintain teeth and restorations through new materials and clinical approaches. Patients may include older individuals, with more complex medical history and oral needs, and/or general health problems with needs for challenging prosthetic management. These patients may be at higher risk of dental diseases and cancer, but with poor access to care. ${ }^{24}$ Patients may also be spread across organisational boundaries including care homes. As well as older individuals, younger patients may require interventions for example, in cases of rapidly progressing tooth wear ${ }^{25}$ (Table 1 , row B). Improving access (Table 1, row A) and reducing oral and dental disease risk (Table 1 , rows $\mathrm{B}$ and $\mathrm{D}$ ), using ongoing, evidence-based prevention, is important. ${ }^{26}$ Based on the definition of
Prosthodontics, ${ }^{19}$ care planning and delivery begins from the initial appointment and diagnoses and continues through on-going maintenance. Managed networks would support maintenance of complex restorations (Table 1, row D).

The multidisciplinary nature of MCNs is particularly relevant to prosthodontics as a broad specialism. MCNs in prosthodontics would involve specialists, patients, carers, general dental practitioners, community practice staff, managers, administrative staff, nurses, technicians, dental therapists and hygienists. MCNs would work alongside other specialisms as well as general medicine and examples are provided in Table 1, row $b$.

The care available would be limited by the costs of clinical specialist services, but would need to be realistic and ostensibly require enhanced payments under the existing remuneration system. MCNs, as a partnership 
of existing bodies, would inevitably reduce costs. In addition, as prosthodontics covers a broad remit of care across networks, funding would benefit from a variety of sources. Relying on solely private sources would increase inequalities and is not preferable. ${ }^{16}$

MCNs create opportunities for highly skilled specialists to input into NHS practice and support, where necessary, care plans and staff education. As most prosthodontic care is provided by non-specialists, a need exists for those with extended skills to provide treatments considered too difficult for GDPs (dentists with special interests) while not complicated enough for specialists. However, there is limited documentation on how to achieve this in prosthodontics. ${ }^{27}$ For newly qualified graduates, MCNs would develop confidence across healthcare professions through an active mentorship process in a community of learning. ${ }^{28}$ Beyond foundation training, such mentorship/training is currently not routine, but has proved beneficial, eg, for early career dentists to promote professionalism and shape career development before independent practice. ${ }^{29}$ It also provides ongoing support in communication with colleagues and patients to help reduce litigation. ${ }^{11}$ In addition, smaller practices might benefit from closer cooperation with clinical resources across networks. ${ }^{22}$ Table 1, row C discusses communication priorities between colleagues and with patients.

In research, large-scale databases of oral and dental health from each MCN could be established and used to evaluate ongoing clinical performances. These may be used to assess access, general medical/oral hygiene screening, restoration/tooth longevity and communication needs based on national and local indicators for clinical excellence.

\section{How can we intervene to improve tooth longevity in an ageing dentition?}

The demands on the dentition and restorations, in an ageing population, and the expectation for restoration longevity, are rising. ${ }^{30}$ Combined with this, there are rapidly advancing materials used in clinical management ${ }^{31}$ and ongoing high quality large-scale research is necessary to inform appropriate interventions. However, there are few large-scale and/or longer-term clinical studies in UK PDC, investigating the longevity of teeth and restorations, ${ }^{32}$ often due to scarce funding. One recent 15 -year prospective unfunded study with tooth and patient outcomes was the first of its kind to investigate the shortened dental arch (SDA) concept for oral rehabilitation in England in PDC. ${ }^{33}$ SDA may be considered as a baseline for access to funded services. Another recent unfunded 50-year study from PDC in England with consistent annual follow-up and patient records exists. ${ }^{32}$ Beyond these, larger scale studies from multiple centres exist, but are often based on historic data ${ }^{34}$ or questionnaire data, ${ }^{31}$ and with a need for patient input to the analyses. One such study, based on 26 million courses of treatment from 1990-2006, was compiled by the Dental Practice Board and released to the research community by the economic and special data service in 2012. ${ }^{34}$ However, this data is fast becoming out-dated and irrelevant with changes to and improvements in materials and technologies. The problems associated with loss of large scale data beyond 2006 is concerning and has been discussed and criticised. ${ }^{35}$

Moving forwards, the use of continuous data collection to provide ongoing large-scale information on the longevity of the dentition and restorations is compelling. An obvious way to build and interpret such large-scale clinical observational data sets from PDC to better inform oral and dental health care delivery is through MCNs. Further recommendations are provided in Table 1, row A and D.

Clinical and laboratory interventions, with industry involvement, have helped investigate materials, eg, to prevent or manage tooth wear and dentine hypersensitivity. ${ }^{36}$ However, further relevant and large multi-centre collaborative investigations are required to target relevant strategies and technologies to benefits oral and dental health.

\section{How can we use and implement digita technologies in dentistry to best improve oral health?}

Digital technologies (Table 1, row E) are likely to play an increasingly relevant role in prosthodontic practice in future. ${ }^{37}$ As mentioned, developments in technologies have fast outpaced service delivery and workforce education in PDC.

Systematic reviews investigating the complete digital workflow in dentistry reveal few randomised control trials (RCTs), with no reported follow-up time, despite fast pace industry led innovations. ${ }^{37}$ Studies are often mono-centred and based in single institutions, perhaps with no direct validity to PDC. Among RCTs that do exist, there are also differences in the outcomes, for example, in terms of mechanical and aesthetic advantages of the final prosthesis. ${ }^{37}$ Important patient outcomes, including satisfaction, are reported in small non-randomised studies, although these are sometimes not wholly in favour of contemporary over conventional practices, ${ }^{38}$ or show little difference on final restoration outcome between techniques. ${ }^{39}$ Similarly, systematic reviews on conventional versus digital impressions again suggest caution over adoption of digital technologies due to the lack of well conducted clinical trials. ${ }^{40}$ There is a need for closer collaboration between manufacturers, academics and clinicians, in order to keep pace with developments, and research the potential and ongoing contribution of new technologies relevant to improving oral health for patients in PDC.

Without definitive evidence-based practice, full digital workflow in prosthetics is questioned. Despite the speed of production and possible cost savings of therapy going forwards there is also a cost implication from the initial equipment. Nonetheless, many dental hospitals and/or some practices have or could adopt partial or full digital workflows. Therefore, possibilities of a fully digital standardised workflow between primary and secondary care exist without a need to purchase digital equipment at all centres. With day-today prosthetic workflow managed digitally, there should be a demand for skilled digital and practical technician roles that complement the innovative workflow system.

Education of digital workflow for the whole dental team may be effectively engaged through academic MCNs. From an education perspective, there are digital benefits to craftbased learning in prosthodontics, by providing constant access to various clinical learning scenarios.

\section{Conclusion}

There is a lack of research from PDC to inform ongoing and often complex clinical prosthodontic practice. With rapidly emerging developments in materials and technologies and increasing challenges and demands within the existing NHS system, including an everageing population with higher expectations, the strain on prosthodontic services in PDC is increasing. We believe a need exists for integrated clinical-academic networks in prosthodontics involving patients, clinicians, industry and academics. These networks would drive research and education for, and promote access to, increasingly complex 
clinical practice to improve oral and dental health. This inevitably will require resources and funding for high quality, research-led clinical practice and dental education for a sufficiently skilled workforce.

\section{Acknowledgements}

The authors wish to acknowledge Professor Jenny Gallagher MBE for her advice in relation to this article.

\section{References}

1. National Institute for Health and Care Excellence. Evidence search. Available online at https://www. evidence nhs uk (accessed 14 February 2019).

2. The James Lind Alliance. Tackling treatment uncertainties together. 2016. Available online at http:// www.jla.nihr.ac.uk/downloads/Introduction-to-the-JLAleaflet.pdf (accessed 14 February 2019).

3. NHS England. Understanding the new NHS. A guide for everyone working and training within the NHS. 2014.

4. Great Britain. Health and Social Care Act 2012: Chapter 7.

5. James Lind Alliance. Priority Setting Partnerships. Available online at http://www.jla.nihr.ac.uk/prioritysetting-partnerships/oral-and-dental-health/ (accessed 14 February 2019).

6. NIHR Clinical and Dental Research Network. Oral and Dental Health Priority Setting Partnership. Available online at https://oralanddentalhealthpsp.wordpress.com (accessed 14 February 2019).

7. National Institute for Health Research. The Top 10 Priorities for Oral and Dental Health Research. 2018.

8. Darzi A. High quality care for all. NHS Next Stage Rev Final Rep 2008; p. 92

9. Darzi A. The Lord Darzi Review of Health and Care: Interim report. Inst Public Policy Res, 2018

10. Barclay S C. Conference report: Shaping the future of restorative dentistry service delivery in the UK. Br Dent J 2018; 224: 296-297.

11. Al Hassan A. Defensive dentistry and the young dentist - this isn't what we signed up for. Br Dent J 2017; 223 . 757-758.

12. Payne D. Persepctives: The dental recruitment crisis. $\mathrm{Br}$ Dent J 2018: 224: 472-475.

13. British Dental Association. NHS dentistry facing recruitment crisis. Press Release. 2018. Available online at https://bda.org/news-centre/press-releases/ nhs-dentistry-facing-recruitment-crisis (accessed 14 February 2019).

14. Nixon P J, Benson R E. A survey of demand for specialist restorative dental services. Br Dent J 2005; 199 : 161-163; discussion 153

15. Sharpe G, Durham J A, Preshaw P M. Attitudes regarding specialist referrals in periodontics. Br Dent J 2007; 202: E11; discussion 218-219.

16. Milsom K M, Jones C, Kearney-Mitchell P, Tickle M. A comparative needs assessment of the dental health of adults attending dental access centres and general dental practices in Halton \& St Helens and Warrington PCTs 2007. Br Dent J 2009; 206: 257-261.

17. O'Selmo E, Collin V, Whitehead P. Associates and their working environment: a comparison of corporate and non-corporate associates. Br Dent J 2018; 225: 425

18. General Dental Council. Registration report - May 2019

19. Driscoll C F, Freilich M A, Guckes A D et al. The glossary of prosthodontic terms. J Prosthet Dent 2017; 117: C1-e105.

20. Campbell S, Tickle M. What is quality primary dental care? Br Dent J 2013; 215: 135-139.

21. NHS England C D O T Guide for Commissioning Specialist Dentistry Services Superseded. 2015; pp 1-91.

22. Skipper M. Managed clinical networks. Br Dent J 2010; 209: 241-242.

23. Willcocks S, Willcocks E. The shift to integrated care in the NHS: implications of the new care models for dentistry. Br Dent J 2019; 226: 319-322

24. Austin R S, Olley R C, Ray-Chaudhuri A, Gallagher J E. Oral disease prevention for older people. Prim Dent Care 2011; 18: 101-106.

25. Olley R C, Sehmi H. The rise of dentine hypersensitivity and tooth wear in an ageing population. Br Dent J 2017; 223: 293-297.

26. Public Health England. Delivering better oral health: an evidence-based toolkit for prevention Summary guidance tables, 2017.

27. Eliyas S, Holsgrove G, Gallagher J E. Education and training in craft skills dentistry. Br Dent J 2019; 226: 795-800.

28. Movahedi S, Eliyas S, Fisher N. Early years postgraduate learning and training in prosthodontic dentistry: 2019 and beyond. Br Dent J 2019: 226: 801-806.

29. Mohamed Osama O, Gallagher J E. Role models and professional development in dentistry: an important resource: The views of early career stage dentists at one academic health science centre in England. Eur J Dent Educ 2018; 22: e81-e87.

30. Olley R C, Sehmi H. The rise of dentine hypersensitivity and tooth wear in an ageing population. Br Dent J 2017 223. DOI: $10.1038 /$ sj.bdj.2017.715
31. Creanor S, Wilson N H F, Jum'ah A A, Brunton P A, Burke F J T. Dental practice in the UK in 2015/2016. Part 3: aspects of indirect restorations and fixed prosthodontics. Br Dent J 2019; 226: 192-196.

32. Olley R C, Andiappan M, Frost P. An up to 50-year follow-up of crown and veneer survival in a dental practice. J Prosthet Dent 2017; DOI: 10.1016/j. prosdent.2017.06.009.

33. Olley R C, Renton T, Frost P M. Observational study investigating tooth extraction and the shortened dental arch approach. J Oral Rehabil 2017; DOI: 10.1111/ joor.12523.

34. Lucarotti P S K, Burke F J T. The ultimate guide to restoration longevity in England and Wales. Part 1: Methodology. Br Dent J 2018; 224: 709-716.

35. Frost $P$ M. Letter to the Editor; criticizing the abolition of statistics at the BSA. Dent Update 2008; 35: 67.

36. Olley R C, Pilecki P, Hughes $\mathrm{N}$ et al. An in situ study investigating dentine tubule occlusion of dentifrices following acid challenge. J Dent 2012; 40: 585-593.

37. Joda T, Zarone F, Ferrari M. The complete digita workflow in fixed prosthodontics: a systematic review. BMC Oral Health 2017; 17: 124.

38. Wismeijer D, Mans R, van Genuchten M, Reijers H A. Patients' preferences when comparing analogue implant impressions using a polyether impression material versus digital impressions (Intraoral Scan) of dental implants. Clin Oral Implants Res 2014; 25: 1113-1118.

39. Sakornwimon N, Leevailoj C. Clinical marginal fit of zirconia crowns and patients' preferences for impression techniques using intraoral digital scanner versus polyvinyl siloxane material. J Prosthet Dent 2017; 118: 386-391.

40. Alikhasi M, Alsharbaty M H M, Moharrami M. Digital implant impression technique accuracy: a systematic review. Implant Dent 2017; 26: 929-935.

41. Austin R S, Olley R C, Ray-Chaudhuri A, Gallagher J E. Oral disease prevention for older people. Prim Dent Care 2011; 18.

42. Moynihan P J, Elfeky A, Ellis I S, Seal C J, Hyland R M, Thomason J M. Do implant-supported dentures facilitate efficacy of eating more healthily? J Dent 2012; 40: 843-850.

43. Ali D A, Brown R S, Rodriguez L O, Moody E L, Nasr M F. Dental erosion caused by silent gastroesophageal reflux disease. J Am Dent Assoc 2002; 133: 734-739.

44. Raibrown A, Giblin L J, Boyd L D, Perry K. Gastroesophageal reflux disease symptom screening in a dental setting. J Dent Hyg 2017; 91: 44-48.

45. Loomans B, Opdam N, Attin Tet al. Severe tooth wear: European Consensus Statement on Management Guidelines. J Adhes Dent 2017; 19: 111-119. 